\title{
Amorphous carbon coatings for the mitigation of electron cloud in the CERN Super Proton Synchrotron
}

\author{
C. Yin Vallgren, G. Arduini, J. Bauche, S. Calatroni, P. Chiggiato, K. Cornelis, P. Costa Pinto, \\ B. Henrist, E. Métral, H. Neupert, G. Rumolo, E. Shaposhnikova, and M. Taborelli \\ CERN, CH 1211, Geneva 23, Switzerland \\ (Received 20 May 2011; published 7 July 2011)
}

\begin{abstract}
Electron cloud buildup is a major limitation for high-energy particle accelerators such as the CERN Super Proton Synchrotron (SPS). Amorphous carbon thin films with low initial secondary electron yield $(\mathrm{SEY} \cong 1.0)$ have been applied as a mitigation material in the SPS vacuum chambers. This paper summarizes the experimental setups for electron cloud monitoring, coating procedures, and recent measurements performed with amorphous carbon coated vacuum chambers in the SPS. The electron cloud measured by dedicated monitors is completely suppressed for LHC-type beams. Even after more than one year's exposure in the SPS with the machine in operation, the coating does not show any increase in the secondary electron yield. The study of coated vacuum chambers for the SPS dipole magnets is in progress; the correlation between electron cloud reduction and pressure rises is not yet fully understood. Some prototypes have already been installed in the accelerator and plans for the implementation of an optimized coating technique are under development.
\end{abstract}

DOI: 10.1103/PhysRevSTAB.14.071001

PACS numbers: 29.27.-a

\section{INTRODUCTION}

In the beam pipes of high-energy proton or positron particle accelerators, an electron cloud (e-cloud) can be generated by residual gas ionization, by photoemission from synchrotron radiation, and by subsequent secondary electron emission via a beam-induced multipactoring process. This effect leads to dynamic pressure rise (electron stimulated desorption), transverse emittance blowup (bunch expansion), thermal load in cryogenic vacuum systems, and beam losses.

A number of possible remedies for the e-cloud have been tested in the beam pipes of various accelerators, e.g., low secondary electron yield (SEY) thin-film coatings, surface conditioning, clearing electrodes, and chamber with grooves or slots. The aim of this work is to find an appropriate solution to eliminate e-cloud in the CERN Super Proton Synchrotron (SPS) in order to make the SPS able to deliver the ultimate beam to the Large Hadron Collider (LHC) and reach maximum luminosity at the collision points.

In the past, TiZrV film coatings have been implemented in the long straight sections of the LHC because this film has an SEY of 1.1 (lower than the SEY threshold value for the e-cloud buildup with the LHC beams [1]) after activation at temperature higher than $180^{\circ} \mathrm{C}$ for 24 hours. However, this film cannot be applied in the SPS since this accelerator cannot be baked. The goal of this work is

Published by the American Physical Society under the terms of the Creative Commons Attribution 3.0 License. Further distribution of this work must maintain attribution to the author(s) and the published article's title, journal citation, and DOI. to find a thin film coating with a reliably low initial SEY, which does not require bakeout and is robust against air exposure. From several earlier studies, carbon is known to have a low SEY [2]. In order to use the existing SPS beam pipes, we chose to produce a thin film coating of amorphous carbon $(\mathrm{a}-\mathrm{C})$ using $\mathrm{DC}$ magnetron sputtering of graphite targets.

In this work, stainless steel liners have been coated with a-C thin films and inserted in the electron cloud monitors (ECM) that are installed in the SPS. In addition, the vacuum chambers of three SPS dipole magnets have been coated. After a description of the experimental setups, the results of the tests performed with LHC types of beam are presented.

\section{SPS EXPERIMENTAL SETUP}

\section{A. Design of the electron cloud monitor}

To detect e-cloud we used the same type of monitors already successfully employed in previous test campaigns $[3,4]$.

The liner (Fig. 1) used for ECM has the same cross section as the vacuum chamber of the SPS dipole magnet. It has a length of $1050 \mathrm{~mm}$ featuring over a length of about $400 \mathrm{~mm}$ a series of holes ( $2 \mathrm{~mm}$ diameter and $6 \mathrm{~mm}$ pitch) providing a geometrical transparency of 7\%. A multistrip detector consisting of 48 copper/kapton strips parallel to the beam axis, with $1.2 \mathrm{~mm}$ pitch, is used to collect the e-cloud current passing through the holes of the grid as a function of the transversal position in the chamber. For each of the 48 channels the current is collected by a series of amplifiers and current to voltage converters with an adjustable gain setting from 2 to 240 . The signal is 


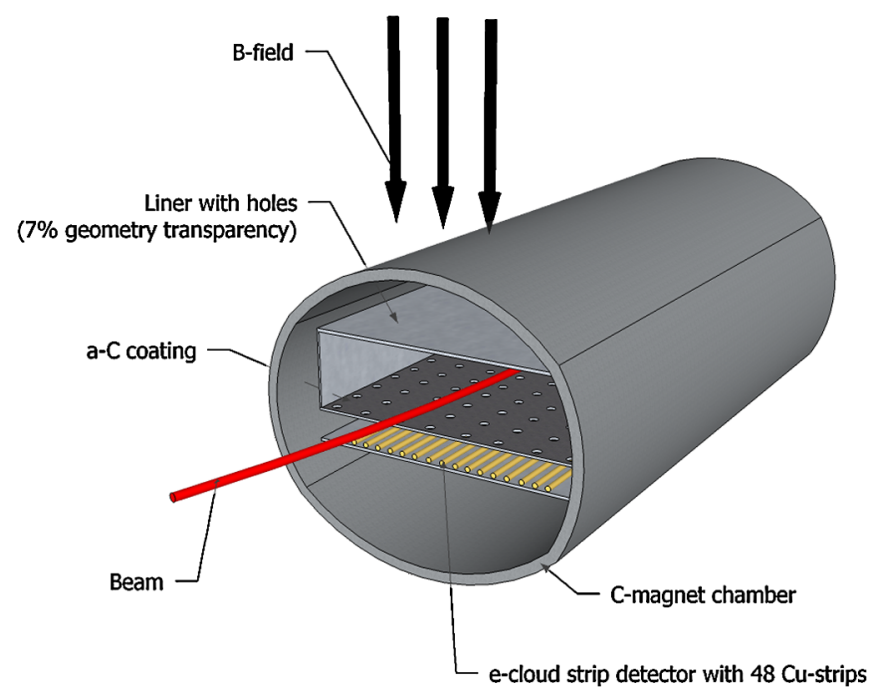

FIG. 1. The schematic view of the liner used for e-cloud monitoring.

averaged over a selectable time span of 10-200 ms. The ECMs and the liners are installed in a round vacuum chamber surrounded by so-called C-magnets, which can apply fields in the range 0 to $2 \mathrm{kG}$. Unless otherwise specified during all the experiments the dipole field was kept at $1.2 \mathrm{kG}$, which corresponds to the SPS injection value.

Four ECMs have been installed in the SPS and can be used at the same operation time. An ECM made of stainless steel (StSt) 316LN, cleaned according to the CERN procedure for cleaning vacuum components, was used as a reference. In general, the StSt liner was exchanged before each machine development (MD) run in order to have a nonconditioned surface as reference (see the different numbering of the StSt liners in Table I). Different a-C coatings have been tested in several MD runs of the SPS, as well as a TiZrV thin film coating (NEG), as listed in Table I.

In addition to the e-cloud monitors, a removable sample was placed in the machine to measure the change of SEY during operation. The removable sample, which spans all the width of the bottom of the SPS vacuum chamber, is $120 \mathrm{~mm}$ long and $60 \mathrm{~mm}$ wide and can be inserted and extracted without breaking the vacuum of the machine. It is hosted in a C-magnet, in a constant magnetic field corresponding to that of the main dipoles' at injection energy $(1.2 \mathrm{kG})$. The field is switched on only during the MD runs. The sample can be transferred to the laboratory under vacuum (typically $10^{-7}$ mbar assured by a turbomolecular pump on or $10^{-4}$ mbar in static vacuum). It is then inserted in an SEY measuring device and in an x-ray photoelectron spectroscopy system for surface analysis (for more details about the measurements, see [5]). In the present experiment a cleaned StSt plate was used as the sample.

\section{B. Setup for the test of a-C coated SPS dipole vacuum chambers}

The schematic view of the test configuration used in sextant 5 of the SPS is shown in Fig. 2. The vacuum chambers of three magnetic benders of B-type (MBB) were coated with an a-C thin film (for more details about coatings, see [5]) and inserted in the machine.

All MBB dipoles used for pressure measurements are assumed to be identical from the point of view of magnetic field and geometry. The conductance from the beam pipe to the respective gauges and ion pumps is identical. The nominal pumping speed of the ion pumps is identical, but some scattering in the effective pumping speed could be present due to unknown differences in the pumps' history. The uncoated dipoles used as reference, which were already present in the SPS, were also vented during installation of the coated dipoles. In most of the experiments the rf shields made of StSt placed in between the dipoles were not coated except in the last period of the tests.

The pressure is read with Penning gauges installed on the pumping port between dipoles. Gauge 51340 monitors the pressure between two uncoated dipoles used as reference whereas gauge 51540 monitors between a-C coated dipoles. Pressure sampling is done at $1 \mathrm{~Hz}$ rate through the standard Prozessvisualisierungs- und Steuerungs-System (PVSS) remote acquisition system. The rate is limited by the data storage speed of PVSS.

\section{Thin film coating}

In this section, a brief description of the coating systems used for producing the liner for the ECMs and the vacuum chambers for the dipole magnets is presented. More details about the thin film coating and the characterization can be found in [5].

The investigated liners are coated by DC magnetron sputtering, using four rectangular graphite rods placed parallel to the liner's main axis. The entire liner is placed inside a cylindrical vacuum chamber surrounded by a solenoid. The first trial of coating the vacuum chamber in the dipole was done with the same magnetic field provided by the dipole itself, which was perpendicular to the cathodes. The power used during coating is also kept limited not to damage the coil. The different coating techniques are compared in Table II. In all the cases presented here, the discharge gas used during the coatings is neon and the thickness of the films is around $100 \mathrm{~nm}$.

\section{EXPERIMENTS}

Various experiments have been performed on the ECMs and in the vacuum chamber of dipole magnets with the LHC-type beams during MD runs in the last three years. For this purpose proton beams of one to four batches of 72 bunches per batch, $25 \mathrm{~ns}$ bunch spacing and intensities of about $10^{11}$ protons per bunch at injection energy of 
TABLE I. The list of used liners in the SPS experiments.

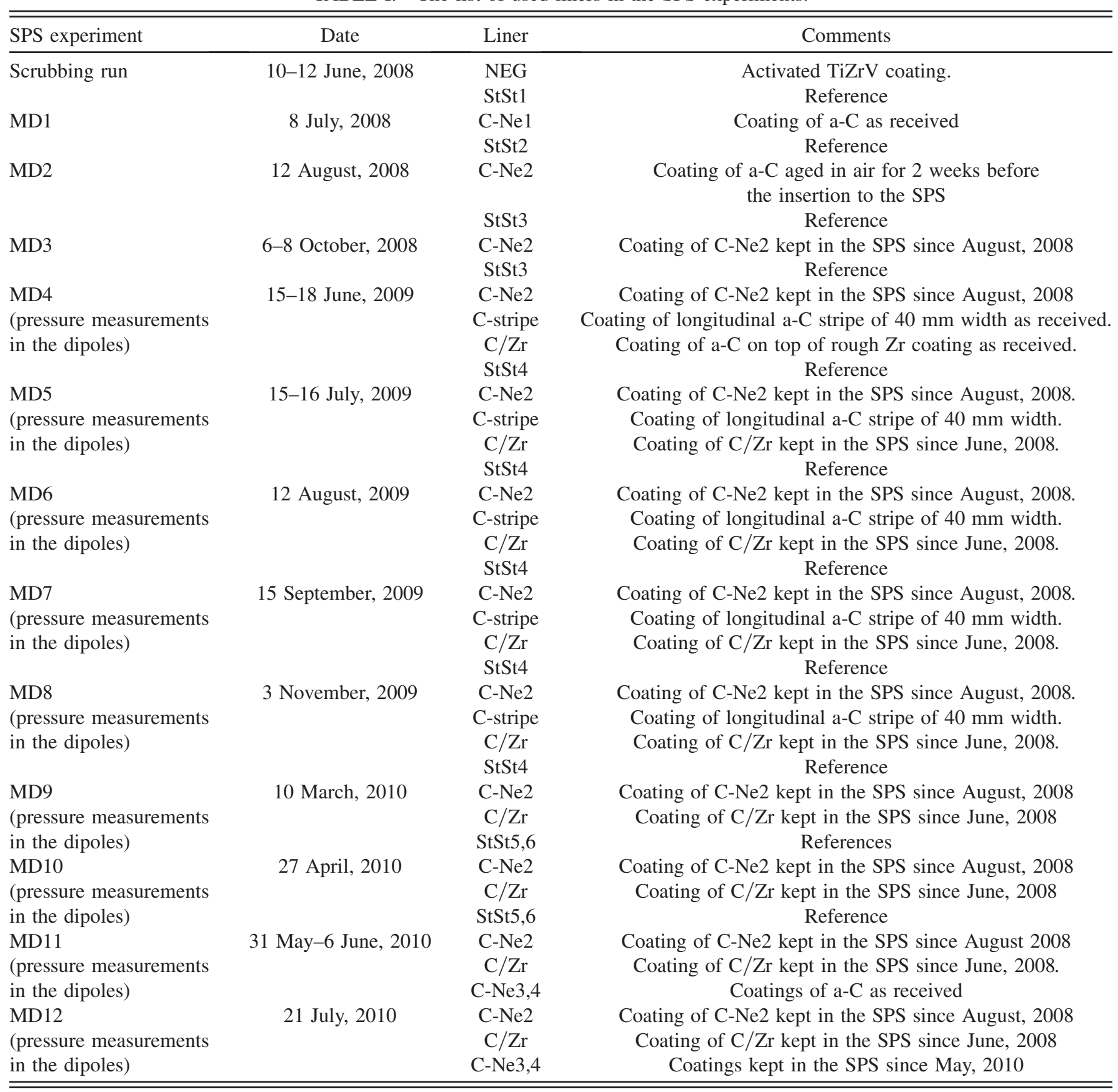

$26 \mathrm{GeV}$ or accelerated to $450 \mathrm{GeV}$ were used. Other time patterns are mentioned explicitly below when necessary. The following experiments were carried out: (i) comparison of e-cloud currents in StSt, NEG, and a-C coated ECM; (ii) observation of ageing effect of a-C coatings (through air exposure during shut-down periods and residence time in the machine vacuum); (iii) measurements of e-cloud effect at various bunch spacings; (iv) measurements of e-cloud currents for a liner coated with a-C only along a central stripe, which is considered to be the relevant region for e-cloud generation; (v) study of magnetic field dependence of measured e-cloud current in ECM; and (iv) measurement of beam-induced pressure rise on the coated and uncoated dipole magnets.

In all cases the beam intensity was measured from the signal of the fast beam current transformer (FBCT). Experiments of microwave transmission in coated and uncoated dipoles were performed in parallel (see [6]).

The inspection of four a-C coated liners and one coated MBB dipole beam pipe after the experiments in the SPS has also been performed. One of the investigated a-C coated liners (C-stripe in Table I) together with the MBB dipole pipe were tested during five MD runs in 2009, with three to four batches of nominal LHC beam accelerated to 


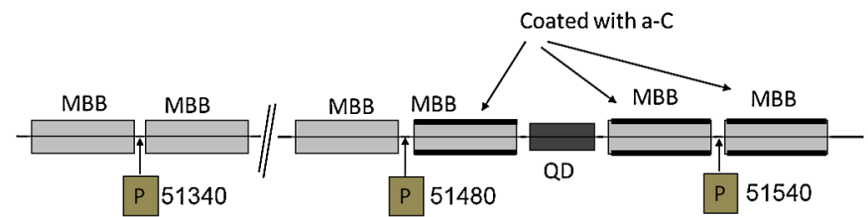

FIG. 2. The schematic setup of the coated and the uncoated magnets in the SPS. MBB: magnetic bender B-type. QD: defocusing quadrupole. $\mathrm{P}$ : Penning gauge and ion pump.

$450 \mathrm{GeV} / c$. Both the liner and the MBB dipole were inserted in the SPS in March 2009, and extracted in February 2010.

\section{RESULTS AND DISCUSSIONS}

For each coated liner the SEY of a witness sample coated simultaneously with the liner was measured in the laboratory. The corresponding curves are reported in Fig. 3. The very first carbon coating, $\mathrm{C}-\mathrm{Ne} 1$, was made of carbon using neon as the discharge gas. The maximum SEY of the witness sample was measured as 0.92 . The second a-C liner was $\mathrm{C}-\mathrm{Ne} 2$, which had been kept in air for two weeks in the coating laboratory prior to installation in the SPS, in order to verify the ageing effect during air exposure. Two further types of liners were inserted to verify the effect of coating roughness and minimum coating area which suppresses e-cloud, respectively. In the first case a double coating with an a-C layer on top of a rough $\mathrm{Zr}$ layer was deposited on a liner and the witness sample showed an SEY of 0.96. In the second case a stripe of a-C coating of width $40 \mathrm{~mm}$ was deposited on the top and bottom faces of the liner along the beam direction and its witness sample had an SEY of 0.92. As a comparison the maximum SEY of StSt cleaned in the same way as the liners is 2.25 and for the activated NEG is 1.1 [1].

\section{A. E-cloud on stainless steel, NEG, and a-C coating}

In Fig. 4, the e-cloud signal normalized to the average beam intensity versus super cycle number in the scrubbing run is shown. Each point represents the time averaged current measured during the supercycle divided by the time averaged FBCT signal. The beam pattern was the standard one with 25 ns bunch spacing without acceleration (injection energy $26 \mathrm{GeV}$ ) and with a supercycle of

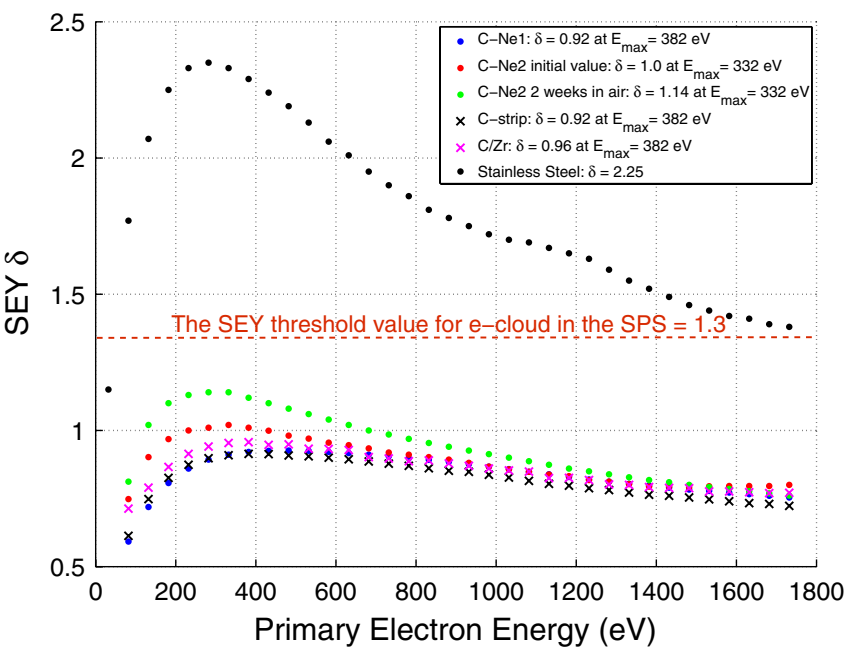

FIG. 3. Overview of the SEY of a-C coatings and StSt reference sample. The threshold value for e-cloud in the SPS with the LHC beams is $1.3[8,11]$.

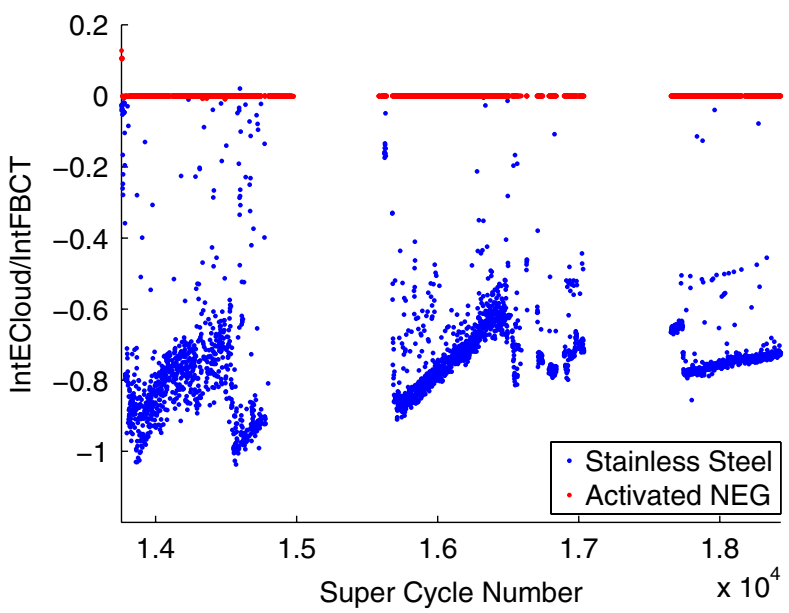

FIG. 4. ECM signals from StSt and NEG liners during the SPS scrubbing run with supercycle length of $43.2 \mathrm{~s}$ : integrated electron current signal (negative values) for each supercycle divided by integrated FBCT signal for each supercycle $\left[\mathrm{nC} / 10^{10}\right.$ protons per bunch] as a function of supercycle number.

43.2 s. The suppression of e-cloud with the activated NEG is expected and was already demonstrated in [7]. It also corresponds well with the threshold of 1.3 predicted by the simulations [8]. The ranking of the intensities of the

TABLE II. Coating of the MBBs was performed in the different conditions than for the liners of ECM.

\begin{tabular}{lcc}
\hline \hline Magnetic field during the sputtering & Liner coatings & MBB coatings \\
Temperature during the sputtering & $\begin{array}{c}\text { The vacuum chambers are inserted in a } \\
\text { solenoid and the magnetic field is parallel to } \\
\text { the cathodes and chamber axis. } \\
\text { The surface temperature can rise to } 250^{\circ} \mathrm{C} .\end{array}$ & $\begin{array}{c}\text { The magnetic field of the dipole is used } \\
\text { and is perpendicular to the cathodes and } \\
\text { chamber axis. }\end{array}$ \\
& $\begin{array}{c}\text { The sputtering power was kept limited } \\
\text { inder to avoid overheating and damaging } \\
\text { of the coil of the dipole. }\end{array}$ \\
\hline \hline
\end{tabular}


e-cloud signals corresponds to the ranking of the SEY measured for the witness samples. The effect of the scrubbing can clearly be seen in Fig. 4. The e-cloud signal for StSt was reduced by a factor 1.6 at the end of the scrubbing run.

In the MD1, the liner $\mathrm{C}-\mathrm{Ne} 1$ was inserted in the ECM. In Fig. 5, the normalized e-cloud signal became $10^{4}$ times lower than that for StSt.

For a clearer view of how much better the a-C coating works for e-cloud suppression compared to StSt, a comparison of SEY measurements done in the lab and the ECM signals measured in the SPS on StSt, C-Ne1, and C-Ne2 liners is shown in Fig. 6. The SEY of both C-Ne1 and $\mathrm{C}-\mathrm{Ne} 2$ is well below the SEY of StSt, as well as the threshold value of e-cloud in MBB, as shown in Fig. 6 (top). In Fig. 6 (bottom), the measured ECM signals on StSt and a-C liners is presented in the base-10 logarithm scale.

\section{B. Observation of ageing effect (in air, in the machine) of carbon coatings}

Figure 5 shows the normalized e-cloud versus time for three different a-C coatings measured in four different MD runs. For the SEY of witness samples deposited simultaneously with the liner, we obtained a value of 1.14 after two weeks of air exposure for C-Ne2, compared to the initial value of 1.0 (see Fig. 3). C-Ne2 with a SEY of 1.14 after two weeks of air exposure showed a higher e-cloud signal than $\mathrm{C}-\mathrm{Ne} 1$ which had a lower SEY of 0.92. On C-Ne2 a visible decrease of e-cloud current occurs after five hours of operation in the SPS during MD run3 (3-4 batches of nominal LHC beam accelerated up to

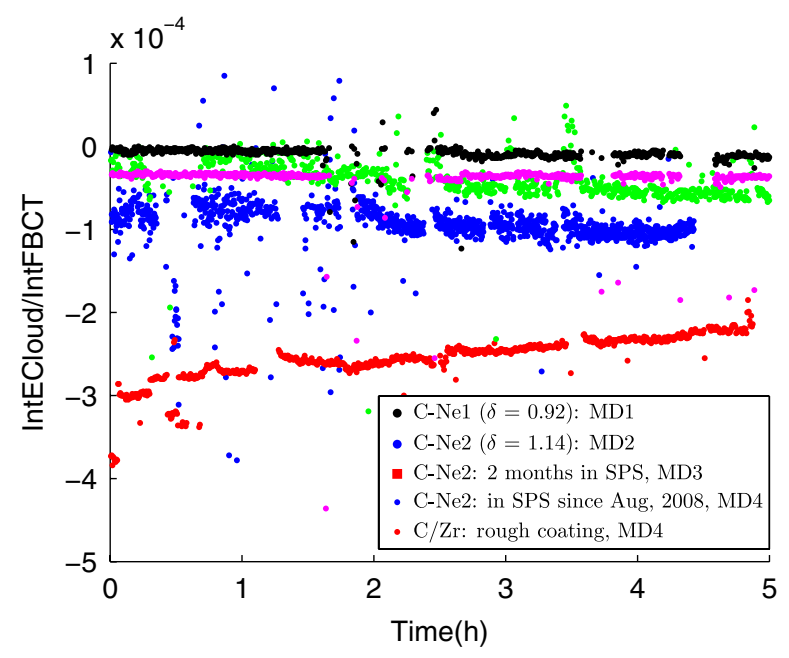

FIG. 5. Normalized e-cloud signals on different a-C coatings from the MD runs. The e-cloud has a magnitude of $10^{-4}$. Integrated electron current signal (negative values) for each supercycle divided by integrated FBCT signal for each supercycle $\left[\mathrm{nC} / 10^{10}\right.$ protons per bunch] as a function of supercycle number.
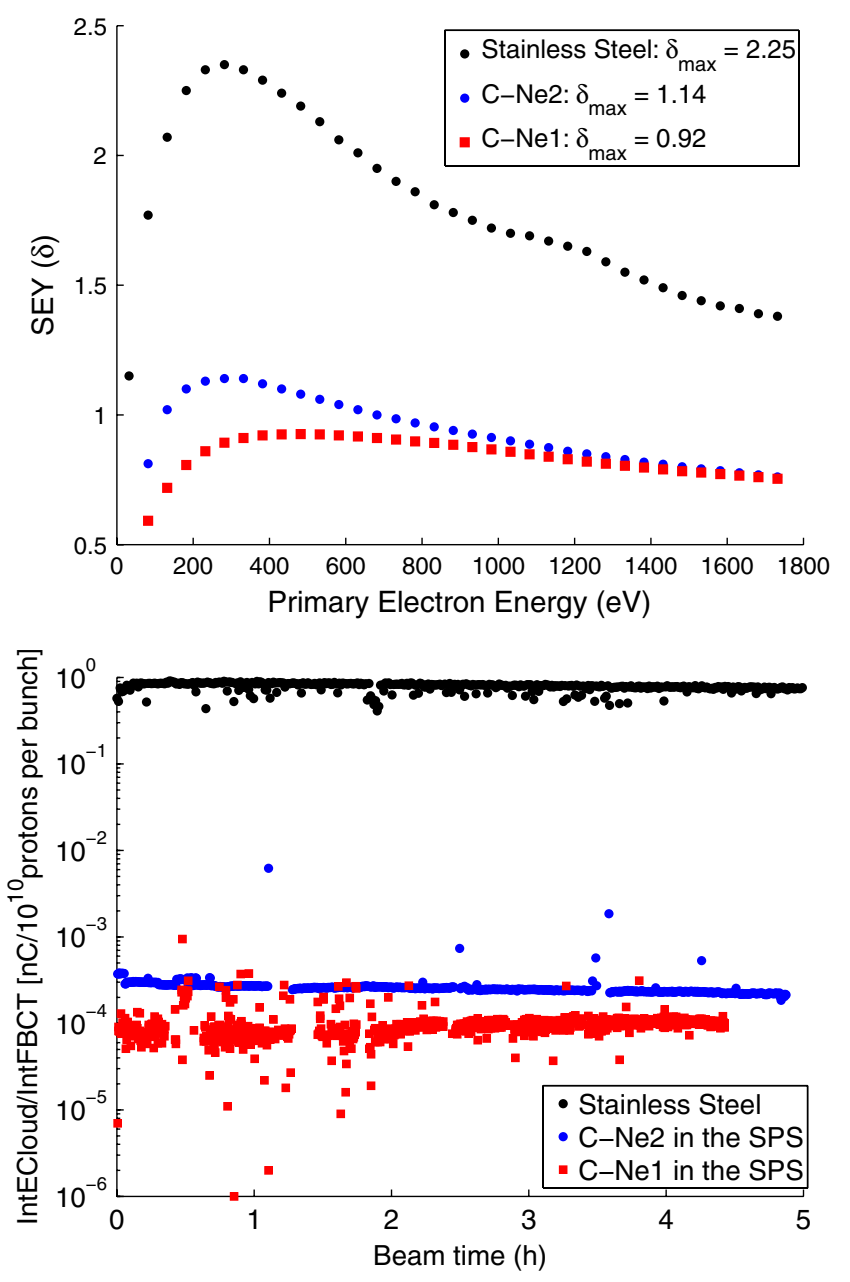

FIG. 6. Top: Comparison of the SEY of StSt and a-C coatings measured in the lab. Bottom: ECM signals from StSt and a-C coatings in the base-10 logarithm of integrated electron current signal for each supercycle divided by integrated FBCT for each supercycle $\left[\mathrm{nC} / 10^{10}\right.$ protons per bunch] as a function of supercycle number.

$450 \mathrm{GeV}$ were used) as shown in Fig. 5. The dose of the electron bombardment on the $\mathrm{C}-\mathrm{Ne} 2$ after five hours of MD was calculated to be about $1.2 \times 10^{-6} \mathrm{C} / \mathrm{mm}^{2}$. After two months in the machine vacuum (low $10^{-8}$ mbar, unbaked) and with normal SPS operation (without LHC-type beams but with the usual beam delivery to CERN neutrinos to Gran Sasso and other fixed target experiments), a new run with LHC-type beam did not reveal any ageing in terms of performance with respect to e-cloud. In addition $\mathrm{C}-\mathrm{Ne} 2$ liner was kept in the SPS during the 2008/2009 winter shutdown and was vented to air for two months. After repumping and operating the accelerator machine during six months, the test with the LHC-type beam exhibited an even stronger reduction of e-cloud on $\mathrm{C}-\mathrm{Ne} 2$. Therefore the liner that remained more than one year in the machine and sustained a prolonged venting during shutdown did not show any sign of deterioration, in contrast it improved its 
performance with respect to e-cloud. Testing of the same liner for a longer term is still in progress.

The visible decrease of e-cloud signal on $\mathrm{C}-\mathrm{Ne} 2$ in three different MDs is not a result of scrubbing effect since no e-cloud is supposed to occur. It could be explained by the reduction of local pressure, which leads to lower residual gas ionization. The pressure from MD 2, 3, 4 has been compared and this confirms a significant improvement by a factor 10 .

\section{E-cloud effect of stainless steel and a-C coating at different bunch spacings}

Two MD sessions using the beam with 50 and 75 ns bunch spacings have been performed to verify the bunch spacing dependence of the e-cloud phenomenon (see Fig. 7). In the first test with a 50 and $75 \mathrm{~ns}$ beam, the ecloud signal for the StSt liner was 5 times lower than the signal with a $25 \mathrm{~ns}$ spacing. For the carbon liner the e-cloud was as low as the detection limit (noise level) of the ECM. After one year of operation, the same StSt liner showed conditioning, demonstrated by the reduction by a factor 2 of the e-cloud at $25 \mathrm{~ns}$ spacing and a much stronger decrease down to detection limit for the 50 and 75 ns spacings. As before no signal was detected on the carbon coated liner. In MD9, the beam with one batch of 36 bunches of $50 \mathrm{~ns}$ bunch spacing was used and the result confirms the outcome of MD3 with the same bunch spacing. These results clearly demonstrate the correlation between e-cloud and bunch spacings and are in excellent agreement with simulations [9].

\section{Minimum coating width to suppress e-cloud in the SPS}

The SEY of the removable StSt sample installed in the SPS was measured after the scrubbing run and MD1. The measurement shows a zone of lower SEY, which is

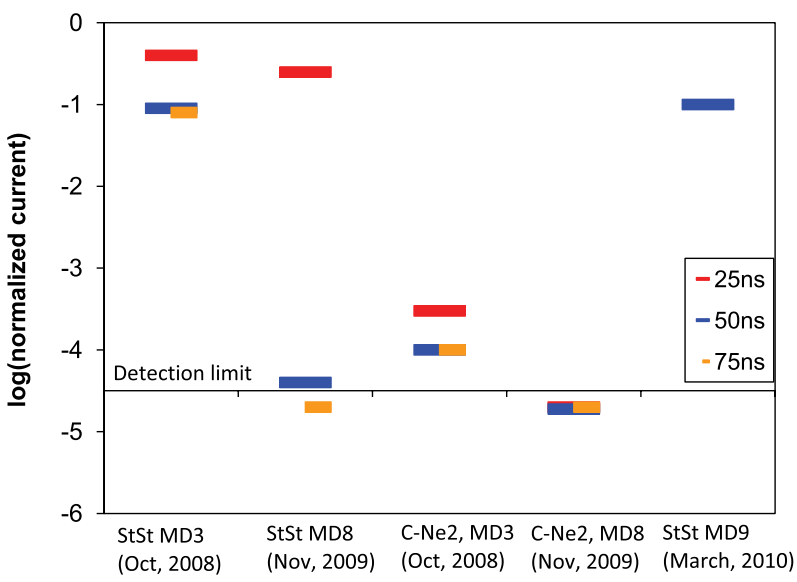

FIG. 7. ECM signals of StSt and a-C coating at different bunch spacings: 25,50 , and $75 \mathrm{~ns}$. In MD8, the e-cloud signal on the $\mathrm{C}-\mathrm{Ne} 2$ liner was well below the detection limit and was considered as noise. conditioned by electron bombardment from the e-cloud in a width of about $40 \mathrm{~mm}$ in the central part (Fig. 8). An additional confirmation that e-cloud occurs only in a central stripe is obtained from the ECM by representing the signal as a function of the various channels of the strip detectors [Fig. 9 (left)]. This was already observed years ago by similar experiments in the SPS $[3,4]$. A liner with a $40 \mathrm{~mm}$ wide carbon coated stripe parallel to beam axis on top and bottom walls has been installed in one of the ECM in order to confirm that such a width is sufficient to suppress e-cloud as seen in Fig. 13(a). In Fig. 9, a comparison of e-cloud signal in the ECM with StSt and carbon stripe liner is shown. After the second batch injection, a slight signal of e-cloud on the carbon strip could be detected only in off center position in two channels of the detector. In this case some e-cloud could develop close to the edge of the carbon stripe on the bare stainless steel surface. This confirms that most of the e-cloud can be suppressed with a

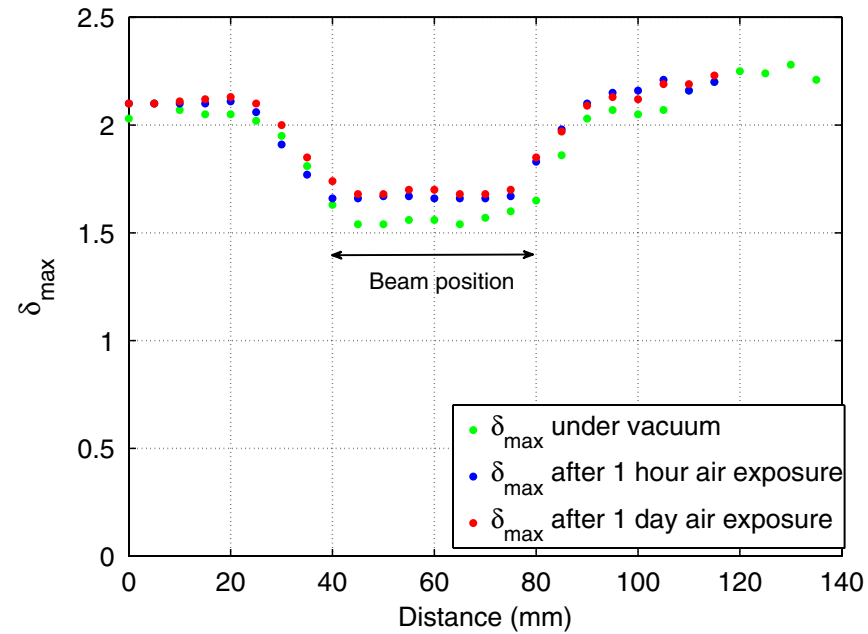

FIG. 8. SEY measurement in the lab on the removable StSt sample transferred from the SPS to the measuring system under vacuum.
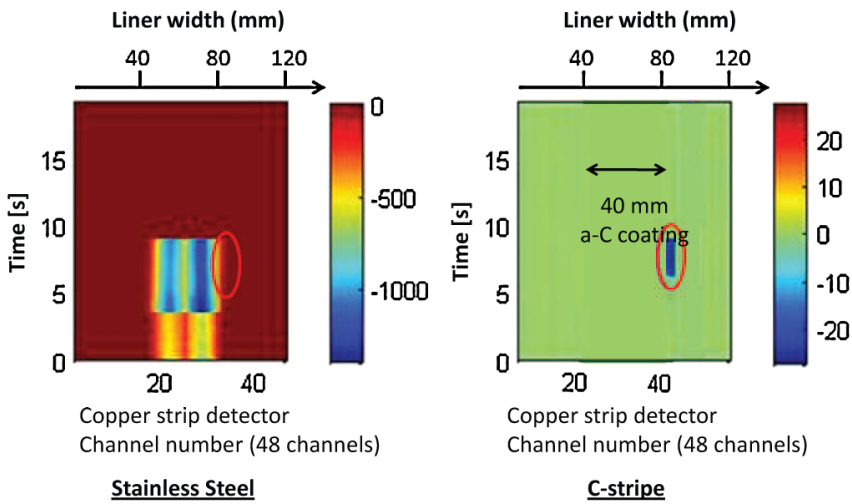

FIG. 9. E-cloud signal measured in the ECMs of StSt and carbon liners with the LHC-type beam of two batches of 72 bunches without acceleration. 
$40 \mathrm{~mm}$ wide coating, but this width of $40 \mathrm{~mm}$ is also quite close to the minimum possible area to be coated for e-cloud mitigation.

We also performed the SEY measurement on the removable StSt sample after one hour and one day air exposure in order to see the ageing effect on the scrubbed StSt. These results also verify the theory that conditioning of beaminduced electron bombardment can be partially reset after air venting.

\section{E. Study of magnetic field dependence of e-cloud current}

Thin dark traces of about $6 \mathrm{~mm}$ along the beam axis have been observed on the top and bottom wall of the dipole chamber made of StSt [see Fig. 10(a)]. A similar pattern has also been observed in the rf shields, which are located between dipoles [see Fig. 10(b)]. The dark traces are believed to be induced by the beam-induced electron bombardment generated by e-cloud. However, the magnetic field between dipoles is known to be very low (about $40 \mathrm{G}$ ), a study of magnetic field dependence of measured e-cloud current was also performed.

The measured charge detected by the ECM with various currents applied on the C-dipole magnet as well as the

(a)

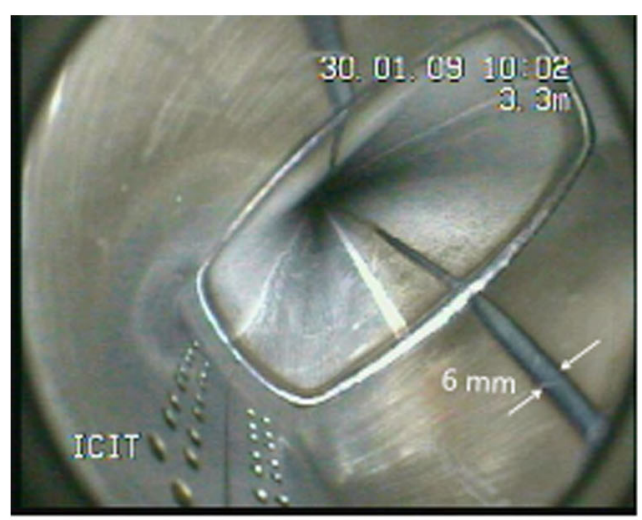

(b)

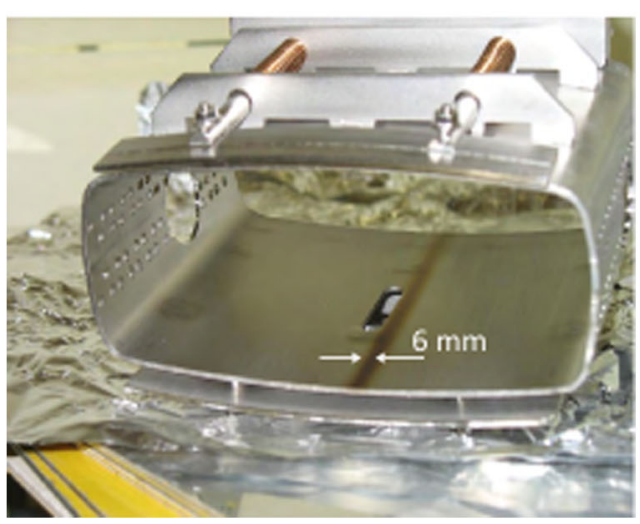

FIG. 10. Thin dark traces along the beam axis in the vacuum chamber of dipole (a) and in the rf shield between dipoles (b).
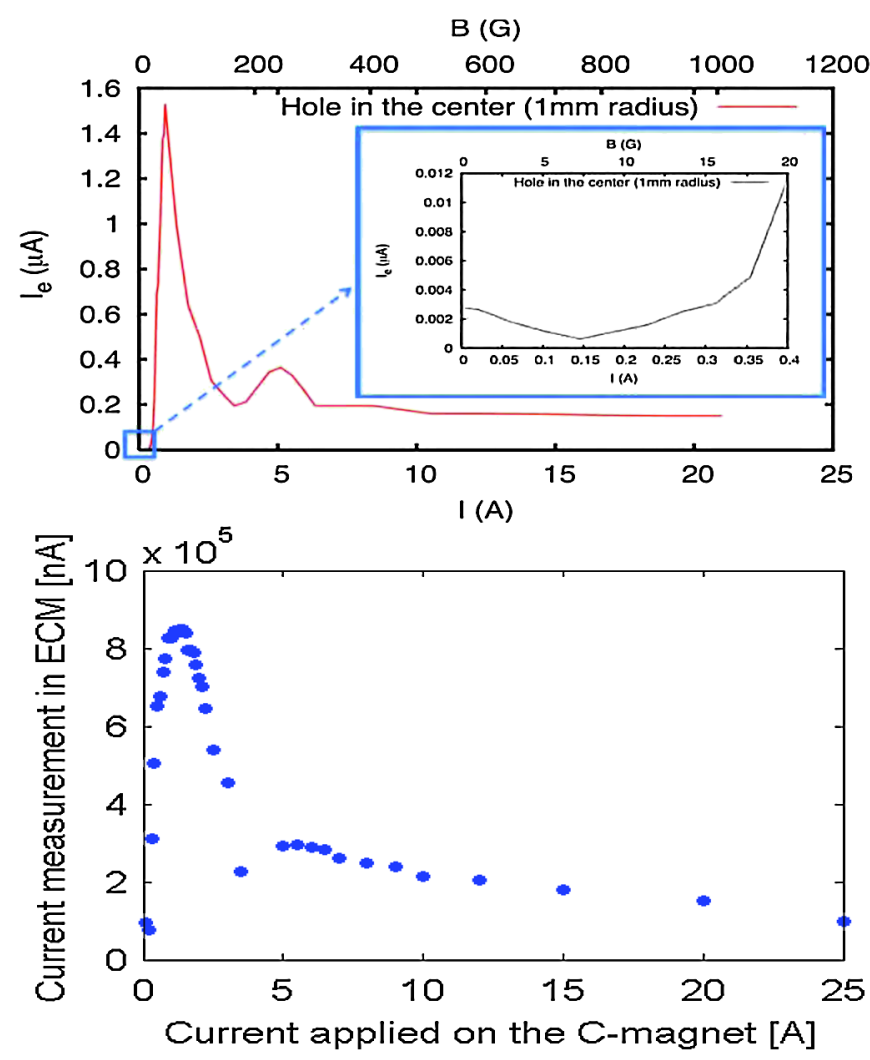

FIG. 11. The current of e-cloud on stainless steel as a function of current applied on the dipole. Top: E-cloud simulation of the total charge into the hole of $1 \mathrm{~mm}$ in radius in the center of the liner. Bottom: Measurement of the total charge detected by ECM. To be noticed: two maxima appear both in the simulation and in the measurements. The first maximum is due to the magnetic field dependence of the transmission of the ECM. At higher magnetic fields the cyclotron radius of secondary electrons is so small that they remain almost constant in the radius of their rotation which is of the same order of magnitude of the holes of the grid (see Fig. 1). This decreases the measured e-cloud at high fields. Therefore the second maximum is on the other hand expected as the real maximum of the measured e-cloud current.

corresponding simulation are shown in Fig. 11. Both show that e-cloud effect can occur even at low magnetic field. One can conclude that $30-50 \mathrm{G}$ are sufficient to provoke the e-cloud with essentially the same intensity as for higher fields. As a consequence, carbon coated rf shields were inserted between the two coated dipoles (for more details, see in Sec. IV F).

\section{F. Pressure rise measurement on the coated and uncoated magnets}

The dipole magnets with coated vacuum chambers were inserted before MD4. The static pressure measured in between two coated magnets was systematically slightly higher than the one between two uncoated magnets which were used as reference (see Fig. 2 for the detailed 
positions). The uncoated reference magnets were vented during insertion of the coated magnets, but had already been in the machine for several years. Therefore the minor difference in the static pressure does not allow us to conclude that the static degassing increases due to the coating.

The dynamic pressure rise measured in the different gauges, indicated in Fig. 2, is shown in Fig. 12 for an LHC-type beam. The pressure rise exhibits the SPS cycle periodicity of $21.6 \mathrm{~s}$. The resolution of the measurement is only $1 \mathrm{~s}$, but the effect of the acceleration ramp is well visible. The scattering in the pressure rise on both coated and uncoated chambers depends on various beam parameters. However, it is obvious that the decrease, if any, due to the coating is not as marked as for the e-cloud signal in the ECM.

A first consideration should be made concerning the maximum effect that we expect on the pressure in the best case when the pressure rise in the coated magnets is completely suppressed. The large gas conductance along the beam pipe limits the reduction of the pressure reading by a factor 4 between the coated dipoles compared to that between the uncoated. Such a strong reduction was never observed.

Therefore two questions arise: whether the e-cloud in the dipoles is or is not suppressed and whether the dynamic pressure rise is or is not related to the e-cloud. Since there is no instrumentation which enables a continuous direct detection of the e-cloud intensity in the dipoles, the only possible comparison is with the simultaneous signal from the ECM. In MD5, a comparison between e-cloud signal on StSt ECM and the measured pressure rise in the dipole magnets indicates that an increase of e-cloud in the StSt monitor is well mimicked by an increase in the pressure rise. It should be noted that at the same time the e-cloud signal in the coated ECM

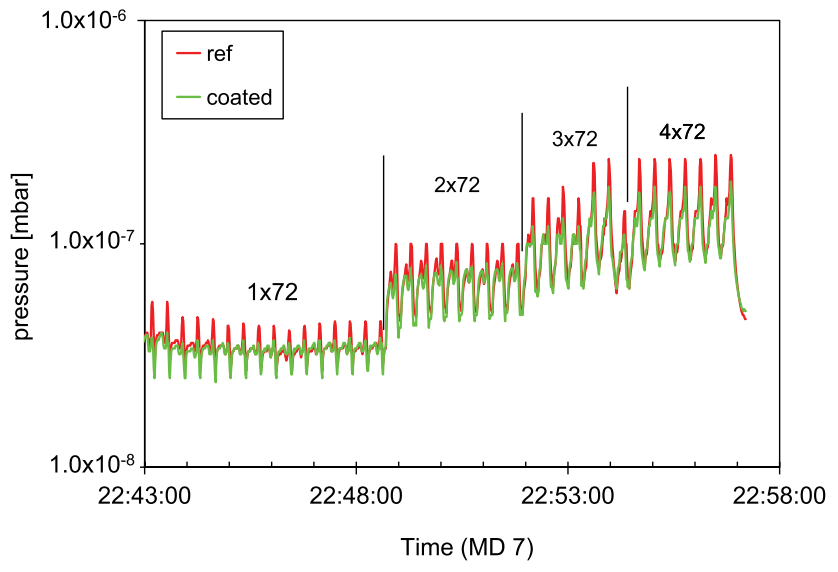

FIG. 12. The comparison of the pressure measurements of the uncoated and coated dipoles. The nominal LHC beam which consisted of one, two, three, and four batches with 72 bunches at $25 \mathrm{~ns}$ spacing and intensity of $10^{11}$ protons/bunch. (not shown) is orders of magnitude lower than for the StSt ECM.

From the experiment with a variable magnetic field in the ECM, we have shown that weak magnetic fields are

(a)

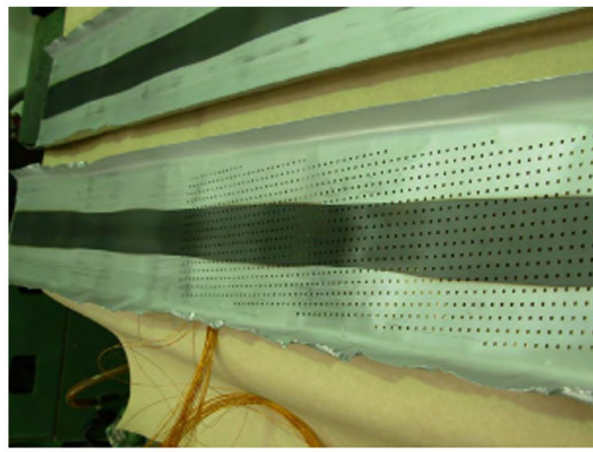

(b)

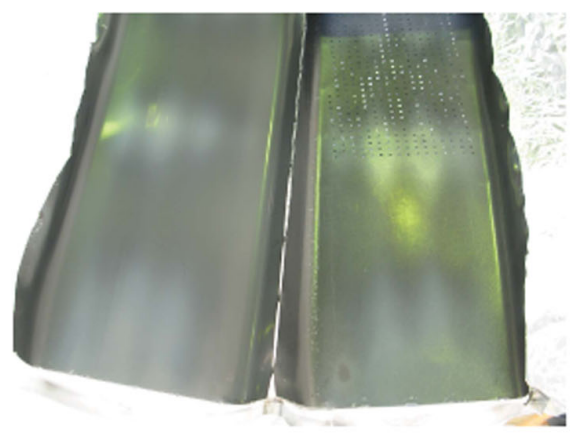

(c)

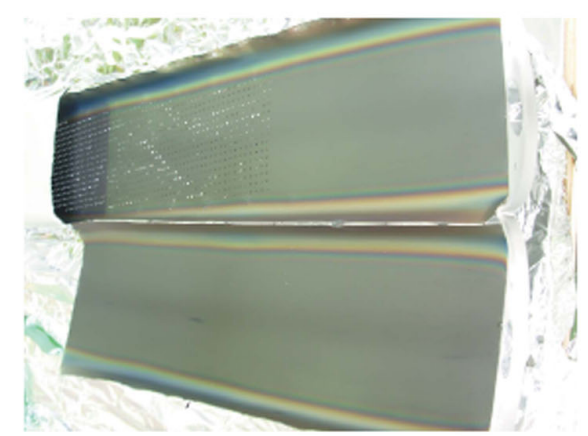

(d)

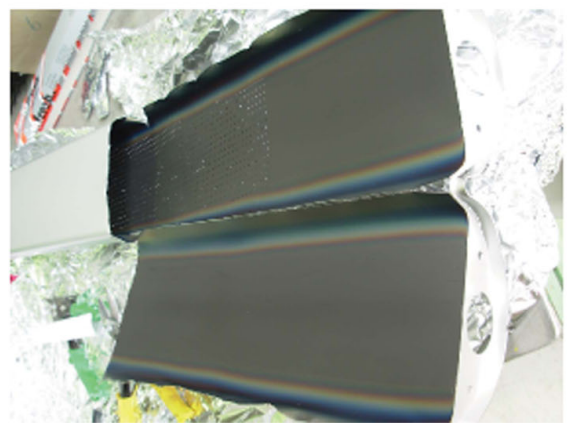

FIG. 13. Inspection of four a-C liners extracted from the SPS. (a) C-stripe, a-C coating used for confirming the minimum of coating width. (b) $\mathrm{C} / \mathrm{Zr}$, a-C on rough $\mathrm{Zr}$ coating. (c) $\mathrm{C}-\mathrm{Ne} 3$. (d) C-Ne4. 
sufficient to provoke the e-cloud phenomenon with essentially the same intensity as for higher fields. Therefore the pressure rise induced by e-cloud could also be strong in between the dipoles, where the fringe field is sufficiently powerful. In order to reduce this gas contribution, carbon coated rf shields were inserted in a further phase between the two coated dipoles. Unfortunately, no evident change was observed in the dynamic pressure rise.

The question remains whether the e-cloud in the dipoles was suppressed or not. The coating of the dipoles was not performed in the same conditions as for the liners. The magnetic field for the magnetron coating of the liners was on an axis parallel to the graphite cathodes, whereas for the dipole the field produced by their coil was used, which is perpendicular to the cathodes. Additional anodes were inserted in the magnets in order to achieve a sufficiently high deposition rate. Another difference between the carbon coating in the liners and the dipoles was the substrate temperature. The liners have a rather bad thermal contact to the surrounding vacuum chamber used during coating and their temperature can rise to $250^{\circ} \mathrm{C}$ during the coating process. Instead the power during the coating is kept low for the dipole magnets in order to avoid overheating and damaging of the insulation of the coils. Samples coated simultaneously and extracted from the dipoles showed an initial SEY close to 1.0, but also exhibited a rather fast increase of the SEY upon air exposure compared to the carbon coatings of liners. Some samples were stored in the pumping ports of the dipoles during one year. Upon extraction they showed an SEY of 1.3, which according to simulations [8] is the theoretical threshold for e-cloud in SPS.

Another mechanism is required to answer the question of what causes the pressure rise. A pressure rise due to ions induced desorption on the walls of the beam pipe can be discarded. At $10^{-8}$ mbar residual pressure the amount of ions produced by the beam $(1 \mathrm{nA})$ is orders of magnitude too low to justify the pressure rise. In addition, such a mechanism would depend linearly on beam intensity and would be totally independent of bunch spacing.

The only mechanism which remains plausible and would depend on beam parameters beyond the beam intensity would be the intensity of proton losses. The local proton loss is unknown and only the integral value is measured and is found to be around $15 \%-20 \%$ for each cycle along the ring.

The relation between the measured pressure rise and e-cloud effect in the dipole magnets is still unclear and more studies are in progress. However, the microwave transmission method proved that the integrated e-cloud density is at least $6 \mathrm{~dB}$ lower in the coated vacuum chamber. Hence, a beneficial effect of this coating was at least indirectly confirmed by other authors [10].
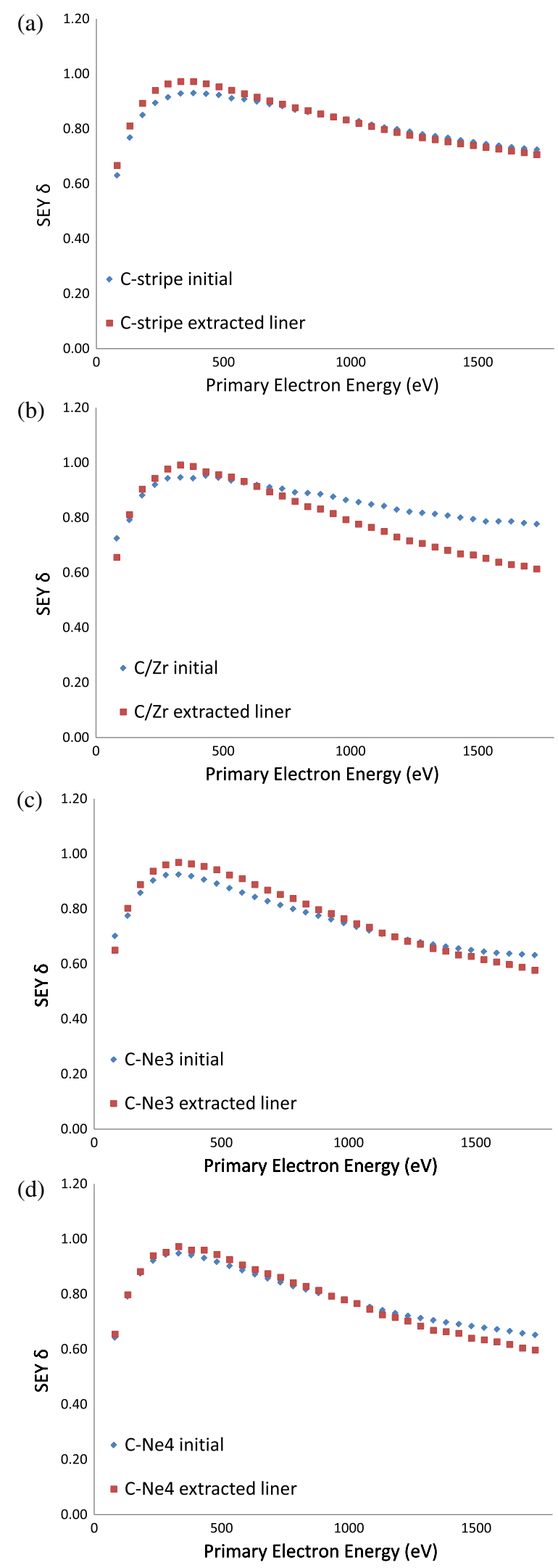

FIG. 14. SEY measurements on four a-C coated liners before and after operation in the SPS. (a) C-stripe, a-C coating used for confirming the minimum of coating width. (b) $\mathrm{C} / \mathrm{Zr}$, a-C on rough $\mathrm{Zr}$ coating. (c) $\mathrm{C}-\mathrm{Ne} 3$. (d) $\mathrm{C}-\mathrm{Ne} 4$. 
(a)

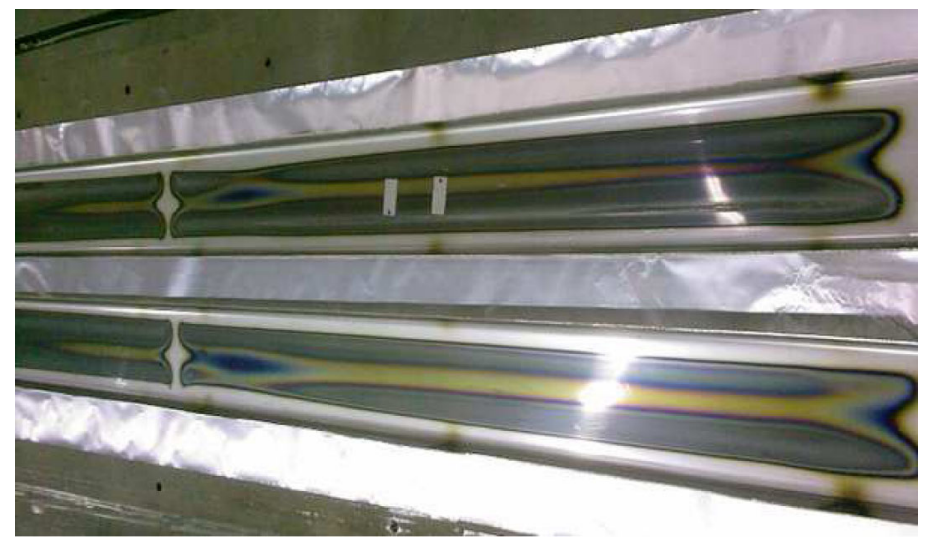

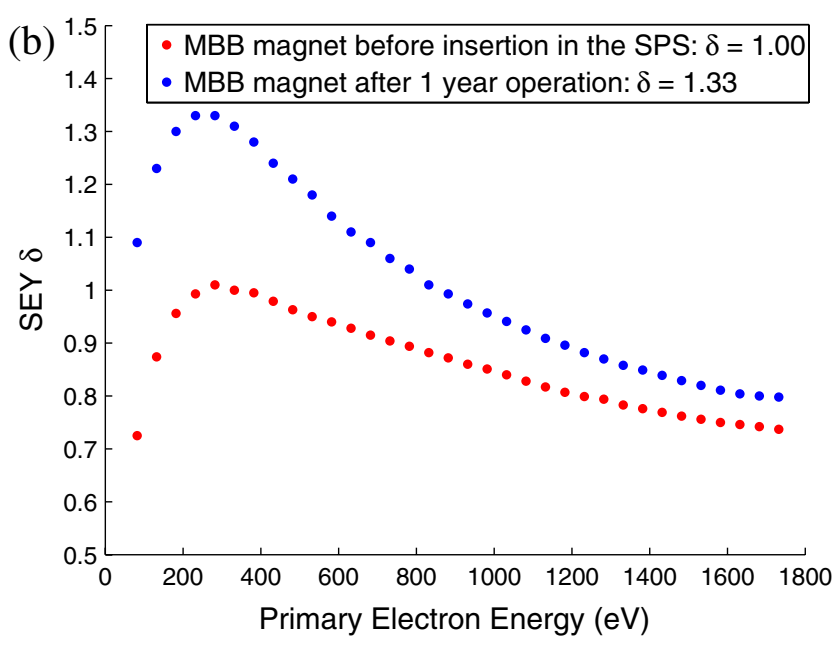

FIG. 15. (a) Inspection of the extracted a-C coated MBB after operation in the SPS. (b) SEY measurement on the coated MBB magnet beam pipe before and after operation in the SPS.

\section{G. Inspection of a-C coated liner and a-C coated MBB magnet}

The recent inspection of four a-C coated liners extracted from the SPS is shown in Fig. 13. These a-C coated liners have all been tested during MD 2-MD 12 runs in 2009, with 3-4 batches of nominal LHC beam accelerated to $450 \mathrm{GeV} / c$. In Fig. 13, a perfectly homogeneous dark coating shows no peeling off and no damage caused by the beam on all four extracted liners after more than one year operation in the SPS. The SEY measurements of these liners after extraction have also been performed in the lab. The increase of the SEY is negligible as shown in Fig. 14.

However, in Fig. 15(a), the coating of the extracted MBB dipole vacuum chamber does not look as homogeneous as the liner seen in Fig. 13, and some part of the chamber is even without coating. In the middle part of the chamber, the coating layer is thin and looks very transparent. This laterally nonuniform coating color indicates differences in thickness and in composition compared to the coating in liners. The SEY measurement of this magnet chamber has also been performed in the lab. The highest SEY occurred in the middle part of the vacuum chamber with a value of 1.33 as shown in Fig. 15(b). This inspection of the coated magnet chamber confirmed that this coating was significantly inferior in quality to the coating in the liners, which gave a complete suppression of e-cloud.

\section{CONCLUSION}

In conclusion the experiments have shown that a complete suppression of e-cloud can be achieved by coating the liners with a thin layer of amorphous carbon, which has a secondary electron yield (SEY) close to 1.0 as measured in the laboratory. The coating does not show an increase of the SEY (ageing) after more than one year's exposure in the SPS vacuum with the machine in operation with beams used for fixed target and a few periods with LHC beam (MD runs). The suppression is confirmed for 25, 50, and $75 \mathrm{~ns}$ bunch spacing. In addition, a coating width of $40 \mathrm{~mm}$ is almost sufficient to suppress completely the e-cloud in these conditions. The inspection of the coated magnet vacuum chamber confirmed that the coating in the magnets was significantly inferior in uniformity indicating the differences in thickness and in composition to the coatings in the liner, which gave a complete suppression of e-cloud.

Future activity will now be focused on modifying the coating system in order to find a solution to coat the dipole magnets with the same quality of coating as in the ECMs. Once the found solution satisfies all criteria, it could be applied to coat most (about 750 beam pipes) of the SPS vacuum chambers.

\section{ACKNOWLEDGMENTS}

We thank all members of the TE-VSC-group and SPSU Study team. The work would not have been possible without their enthusiasm and help.

[1] P. Chiggiato and P. C. Pinto, Thin Solid Films 515, 382 (2006).

[2] J. M. Ripalda, I. Montero, L. Vázquez, D. Raboso, and L. Galán, J. Appl. Phys. 99, 043513 (2006).

[3] G. Arduini, P. Collier, B. Dehning, G. Ferioli, B. Henrist, L. Jensen, J. M. Jimnez, J. M. Laurent, R. Rumolo, K. Weiss, and F. Zimmermann, in Proceedings of the 8th European Particle Accelerator Conference, Paris, 2002 (EPS-IGA and CERN, Geneva, 2002).

[4] E. Shaposhnikova, G. Arduini, J. Axensalva, E. Benedetto, S. Calatroni, P. Chiggiato, K. Cornelis, P. C. Pinto, B. Henrist, J. Jimenez, E. Mahner, G. Rumolo, M. Taborelli, and C. Y. Vallgren, in Proceedings 
of the 23rd Particle Accelerator Conference, Vancouver, Canada, 2009 (IEEE, Piscataway, NJ, 2009).

[5] C. Y. Vallgren, A. Ashraf, S. Calatroni, P. Chiggiato, P. C. Pinto, H. Marques, and H. Neupert, in Proceedings of the IPAC'10 Conference, Kyoto, Japan (ICR, Kyoto, 2010).

[6] S. Federmann, F. Caspers, E. Mahner, P.C. Pinto, M. Taborelli, B. Salvant, and C. Y. Vallgren, in Proceedings of the IPAC'10 Conference, Kyoto, Japan (Ref. [5]).

[7] J. Jimenez, G. Arduini, V. Baglin, P. Collier, G. Ferioli, B. Henrist, N. Hilleret, L. Jensen, B. Jenninger, J. Laurent, A.
Rossi, K. Weiss, and F. Zimmermann, in Proceedings of the 20th Particle Accelerator Conference, Portland, OR, 2003 (IEEE, New York, 2003).

[8] G. Rumolo, in http://indico.cern.ch/getFile.py/access? contribId $=2 \&$ session $I d=0 \&$ resId $=0 \&$ materialI $d=$ slides $\&$ confId=62873 (2009).

[9] G. Rumolo, in https://paf-spsu.web.cern.ch/pafspsu/ meetings/2007/M20-11/GR-SPSU5.pdf (2007).

[10] S. Federmann, F. Caspers, and E. Mahner, Phys. Rev. ST Accel. Beams 14, 012802 (2011).

[11] G. Rumolo, F. Ruggiero, and F. Zimmermann, Phys. Rev. ST Accel. Beams 4, 012801 (2001). 